operating hours' should be acceptable to large departments such as theirs but would scarcely be tolerable to the majority of NHS histopathologists, often working single-handed and giving cover to more than one hospital. Conceding that toluidine blue and aqueous mounting media save minutes, time, despite the frenetic age we live in, is not now so critical and the extra couple of minutes required to complete a rapid haemalum and eosin preparation would be acceptable to any surgeon. The advantages are obviously a permanent slide and an easier adaptation from paraffin to frozen sections for the inexperienced. The book would have been improved by beginning each section with a discussion of the important pitfalls and difficulties: florid sclerosing adenosis versus carcinoma of the breast, viral lymphadenitis versus malignant lymphoma, chronic pancreatitis versus sclerosing carcinoma of the pancreas, proliferative fibromatosis versus fibrosarcoma, and juvenile melanoma in the adult are a few examples that readily spring to mind. Granted that many of these diagnostic difficulties can be gleaned from the illustrations and their captions they nonetheless require special emphasis if therapeutic disasters are to be avoided by the enthusiastic, inexperienced histopathologist. The authors are to be congratulated on using their routine toluidine blue sections for illustration, showing them warts and all and resisting the temptation to indulge in window dressing. With few exceptions the colour reproductions are of high quality though I would not recommend a practice of picture matching to the histopathologist faced with a difficult frozen section.

$$
\text { W. B. ROBERTSON }
$$

Cytology Automation. Proceedings of Second Tenovus Symposium Edited by D. M. D. Evans. (Pp. 275; illustrated. £3.00). Edinburgh and London: E. \& S. Livingstone Ltd. 1970.

The proceedings of the 1968 conference published two years later is still interesting reading although many of the hopes expressed have not been realized. The importance of the conference and the report is that the participants were interested in developing the subject from the 'hack screening' which so often produces the moribund, frustrated departments of cytology into active, interested laboratories continually developing the subject. First to establish the accepted criteria for abnormality, then to find methods of scanning for such cells by any automated method is an admirable goal and should be pursued if any progress is to be made in the understanding of cervical cytology. None of the methods have so far borne much fruit.

A. G. SIGNY

Laboratory Diagnosis of Kidney Disease Edited by F. W. Sunderman and F. W. Sunderman, Jr. (Pp. xv + 587; illustrated. £12.60). St Louis, Missouri: Warren H. Green, Inc. London: Adam Hilger Ltd. 1971.

In the preface this book is described as containing '... the edited proceedings of an Applied Seminar on the Laboratory Diagnosis of Kidney Diseases held in Washington, D.C. under the auspices of the Association of Clinical Scientists'. The 50 chapters in this book presumably therefore comprise the papers read at that seminar and as such the title is misleading. The material covered ranges widely over the fields of normal renal function and structure to the biochemical and histopathological changes associated with kidney disease. However, as with the published proceedings of any symposium, the subject implied by the title is not covered comprehensively and there are many notable gaps. The more detailed information which is in this book is readily available in the standard texts on renal physiology, histopathology, and chemical pathology, and, therefore, especially at the price, the book cannot be recommended.

M. R. WILLS

Interpretation of Diagnostic Tests. A Handbook Synopsis of Laboratory Medicine By Jacques Wallach. (Pp. xxxii $+441 ; 5$ figures; 60 tables. $£ 3 \cdot 00$ ). Boston: Little, Brown and Co. London: J. and $A$. Churchill Ltd. 1970.

Despite disclaimers in the preface this synopsis contains many esoteric or outmoded tests, a number of surprising omissions, and some inaccuracies. It will not 'make it simpler for the physician to select the most useful laboratory test' but is a powerful argument for an interpretative clinical pathology service.

A. E. GREEN

The Blood Supply of Bone By Murray Brookes. (Pp. ix $+338 ; 219$ Figures.

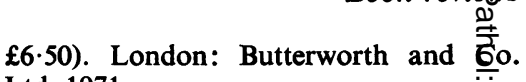
Ltd. 1971.

This book is to be welcomed as a brief $\frac{\overrightarrow{\bar{N}}}{\text { कूnd }}$ well illustrated account of the subject. based on a review of the literature and author's extensive personal research. $\overparen{\overparen{D}}$

It is essentially an anatomist's account of the subject. There are chapters haemodynamics and on other physiological aspects of the intra-osseous circulation, but the main emphasis - is given to the morphological study of blesd vessels when visualized using microscosic, radiographic, and other injection teehniques.

Most of the book describes the normal anatomy of the larger and smaller blopd vessels in bone, but there are also binef sections on the role of vascular factors in disease processes, including occlusive vascular disease, avascular necrosis - of bone, fracture repair, and osteoarthritis. The author regards circulatory factorf as having a central position in the controf of bone growth and renewal, and this point of view is elaborated in the final sectiobुof the book.

\section{Intestinal Absorption and its Derangements}

The proceedings of the symposiun on 'Intestinal absorption and it: derangements' organized by The Royal College of Pathologists and delivered in London in February 1971 will be published on 21 Octobep as a supplement to the Journat. of Clinical Pathology, price $£ 2 \cdot 0 \overline{\mathrm{Q}}$ including postage. Members of Ther Royal College, members of the Association of Clinical Patholor gists, and subscribers to the Journay of Clinical Pathology will be able to buy the supplement at the concef sionary price of $£ 1.75$, includin $\$$ postage. Copies may be obtained from the Publishing Managerp BMA House, Tavistock Square London WC1H 9JR. 